Proceedings of the Symposium on Synchrotron Crystallography, Krynica, Poland 2001

\title{
Design of a 5-Station Macromolecular Crystallography Beamline at MAX-Lab
}

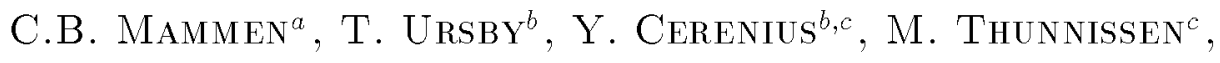 \\ J. Als-Nielsen ${ }^{a}$, S. Larsen ${ }^{d}$ AND A. LILJAS ${ }^{b}$ \\ ${ }^{a}$ Niels Bohr Institute, Ørsted Laboratory, University of Copenhagen \\ Universitetsparken 5, 2100 Copenhagen, Denmark \\ ${ }^{b}$ MAX-lab, Lund University, P.O. Box 118, 22100 Lund, Sweden \\ ${ }^{c}$ Molecular Biophysics, Center for Chemistry and Chemical Engineering \\ Lund University, P.O. Box 124, 22100 Lund, Sweden \\ ${ }^{d}$ Centre for Crystallographic Studies, Department of Chemistry \\ University of Copenhagen \\ Universitetsparken 5, 2100 Copenhagen, Denmark
}

\begin{abstract}
A beamline for macromolecular crystallography is under construction at the Swedish synchrotron light source MAX-lab at Lund University in a collaborative effort between Denmark and Sweden. Of the $7 \mathrm{mrad}$ horizontal wiggler fan emitted from the new superconducting multipole wiggler, the central $2 \mathrm{mrad}$ will be used and split in three parts. The central $1 \mathrm{mrad}$ will be used for a tunable station optimised for multi-wavelength anomalous diffraction experiments and on each side of the central fan there will be two fixed wavelength stations using different energies of the same part of the beam. These in total five stations can be used simultaneously and independently for collecting diffraction data.
\end{abstract}

PACS numbers: 07.85.Qe, 87.14.Ee, 87.14.Gg

\section{Introduction}

A three-dimensional model of a protein at the atomic level provides a wealth of information. In addition to showing the overall folding topology, it provides the basis for a detailed understanding of its biological function [1]. X-ray crystallography plays a central role in the development of structural biology, as the major experimental method for determining high-resolution models of macromolecules $[2,3]$. The rapid growth of determined biological macromolecular structures [2] and the expected accelerated growth due to the advent of structural genomics projects 
[4] are expected to have a large impact on e.g. pharmaceutical development [5, 6]. Another example of the technological interest in structural biology is the potential importance of high-resolution structural determinations for the development of enzymes with new properties [7].

$\mathrm{X}$-ray synchrotron radiation provides many advantages compared to conventional X-ray sources, due to its high intensity and highly collimated radiation with wavelength tunability, increasing the accuracy and ease of structure determinations and extending the applicability of X-ray crystallography to more difficult projects that are often also the more interesting ones. The use of synchrotron radiation has played a fundamental role in the possibility to tackle more difficult problems as well as in the increased structure determination rate [8]. More than $90 \%$ of the publications in macromolecular crystallography in 2000 were based on measurements with synchrotron radiation [9].

Progress in molecular biology and computational techniques together with the increased availability of dedicated synchrotron beamlines has contributed to a rapidly increased use of the multi-wavelength anomalous diffraction (MAD) technique for structure determinations [10]. In a MAD experiment data is collected at several wavelengths around an absorption edge of one of the elements in the macromolecule. By exploiting the anomalous scattering the so-called crystallographic phase problem can be solved and complete structural models be determined. In a majority of the cases Se in the form of Se-methionine is the anomalous scatterer with its $K$ absorption edge at $0.98 \AA(12.7 \mathrm{keV})$. The MAD technique also holds the promise of being suitable for the increased through-put desired by structural genomics projects [11] though the single-wavelength anomalous diffraction (SAD) technique might prove more suitable in some cases [12]. In SAD experiments the anomalous signal is used as in MAD experiments but data is collected at only one wavelength, chosen to optimise the anomalous signal.

The demand for synchrotron beam time is not only related to the possibility of exploiting the wavelength tunability required by MAD and SAD experiments, but also to a large extent due to the improved data quality at synchrotron beamlines compared with laboratory sources. Large structures such as viruses and large cellular complexes are examples where synchrotron beam time is a necessity, but this is true also in many other cases. Structure determinations of potential drug targets in complexes with drug candidates is one example where abundant beam time with rapid access can play a crucial role.

In order to do measurements with synchrotron radiation one has to apply for beam time well in advance at one of the available synchrotron light sources around the world. The beam time available is not sufficient to meet the increasing demands caused by the rapid growth of structural biology. The MAX II synchrotron radiation facility at MAX-lab, Lund, is a $1.5 \mathrm{GeV}$ storage ring [13] with eight straight sections for insertion devices that can operate up into the hard X-ray regime. It is therefore a very attractive resource for researchers in bio-crystallography. The 
purpose of the here described beamline is to further exploit this potential by increasing the available beam time and to extend the capacity to MAD and SAD experiments. The only crystallography beamline in Scandinavia so far, I711 at MAX-lab [14], is only partly dedicated to macromolecular crystallography and is only slowly tunable making it non-suitable for MAD experiments.

The beamline has been named Cassiopeia after the five-star constellation where the Danish astronomer Tycho Brahe, living not far from Lund, discovered a supernova in 1572 .

The Cassiopeia-911 beamline [15] is a unique facility with five experimental stations at one beamline. It is the result of a collaboration between Danish and Swedish researchers in the Øresund region, from Lund and Copenhagen University. The economical basis for the construction of the beamline comes from a grant to the Department of Molecular Biophysics, Lund University, from the Knut and Alice Wallenberg Foundation, a grant to the Centre for Crystallographic Studies, University of Copenhagen, from The Danish Biotechnology Instrument Center and from a consortium formed by the two pharmaceutical companies AstraZeneca and Novo Nordisk A/S. The beamline is also supported by the Swedish Research Council through MAX-lab.

\section{The new MAX-wiggler}

The source for the beamline is a new cold-bore superconducting multipole wiggler designed and built at MAX-lab [16]. Some parameters describing the wiggler are found in Table $\mathbf{I}$.

TABLE I

Some parameters describing the superconducting wiggler.

\begin{tabular}{l|rl}
\hline \hline Number of poles & 49 & \\
Wiggler period & 6.1 & $\mathrm{~mm}$ \\
Length of wiggler, $L_{\text {wiggler }}$ & 1512 & $\mathrm{~mm}$ \\
Peak field & 3.54 & $\mathrm{~T}$ \\
Deflection parameter, $K$ & 21.2 & \\
Electron beam energy, $E_{\mathrm{e}}$ & 1.5 & $\mathrm{GeV}$ \\
Electron beam current & 250 & $\mathrm{~mA}$ \\
Critical energy & 5.2 & $\mathrm{keV}$
\end{tabular}

The wiggler radiation is emitted in a divergent cone as illustrated in Fig. 1. The horizontal and vertical opening angles are given by [17]:

$$
K / \gamma=7 \operatorname{mrad}(\mathrm{h}), \quad 1 / \gamma=0.34 \operatorname{mrad}(\mathrm{v})
$$

where $\gamma=E_{\mathrm{e}} / m_{\mathrm{e}} c^{2}$, with $m_{\mathrm{e}} c^{2}$ being the electron rest energy (see also Table I). Due 
to the typical length of wigglers in the order of one or a few meters, the virtual source size increases significantly at large viewing angles. Since the maximum acceptable photon beam emittance at the sample is around $1 \mathrm{~mm} \cdot \mathrm{mrad}$, only the central part of the wiggler fan will be used for the experimental stations.

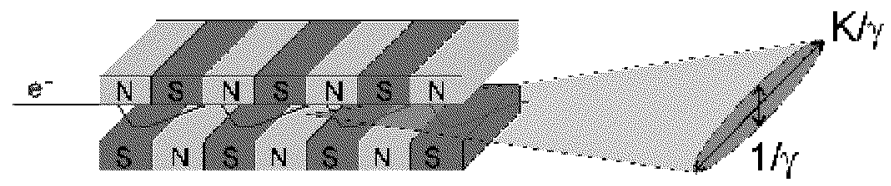

Fig. 1. Schematic drawing of the magnetic array of a wiggler with its radiation cone.

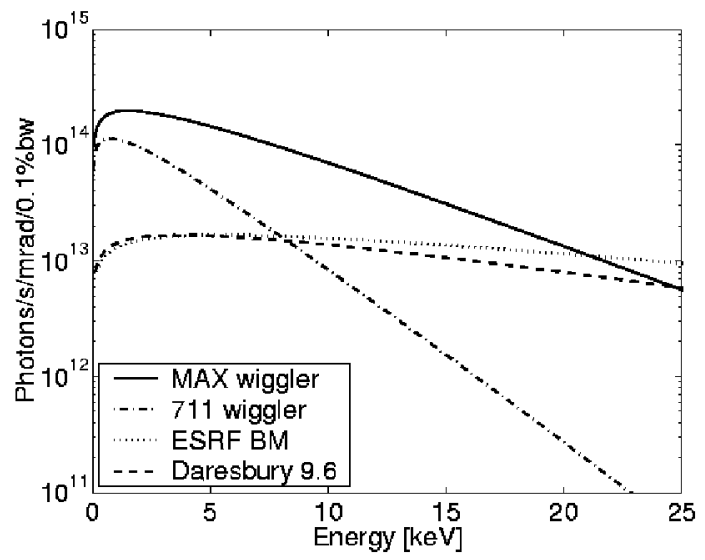

Fig. 2. Comparison of the calculated flux from the new MAX-wiggler with fluxes from the MAX-lab I711 wiggler, the ESRF bending magnet beamline BM14, and the 9.6 beamline at SRS Daresbury. The flux is given per horizontal opening angle.

The calculated flux of the source is plotted in Fig. 2 and compared with the source flux of some existing beamlines for macromolecular crystallography. Wavelengths around $1 \AA(12.4 \mathrm{keV})$ are suitable for most macromolecular structure determinations though wavelengths at longer or shorter wavelengths can be required e.g. for MAD experiments. The range $0.7-1.8 \AA(7-18 \mathrm{keV})$ covers the requirements of most experiments. The flux of the new MAX-wiggler in that wavelength range is indeed comparable with the shown existing beamlines, though the brilliance of $3 \times 10^{15}$ photons/( $0.1 \% \mathrm{bw} \mathrm{mrad} \mathrm{mm}^{2}$ ) ( $\mathrm{bw}=$ band width) is up to five orders of magnitude inferior to undulator beamlines at higher-energy storage rings such as ESRF [18]. This means that the Cassiopeia-911 beamline will have flux values comparable to these sources but will not reach the same small focal spot sizes or high collimation of the radiation. The total power of the wiggler is of the order of $5 \mathrm{~kW}[16]$. 


\section{Concept of the beamline}

The central $1 \mathrm{mrad}$ of the radiation cone, Fig. 1, will be used exclusively for an energy-tunable station, optimised for MAD experiments, and the adjacent $0.5 \mathrm{mrad}$ on each side will be used for four fixed wavelength side stations diffracting horizontally away from the synchrotron beam, as illustrated in the "top view" in Fig. 3. The wavelengths of the side stations are chosen in part to cover some important absorption edges used for SAD experiments and in part to give the highest quality beam for experiments not related to anomalous scattering.

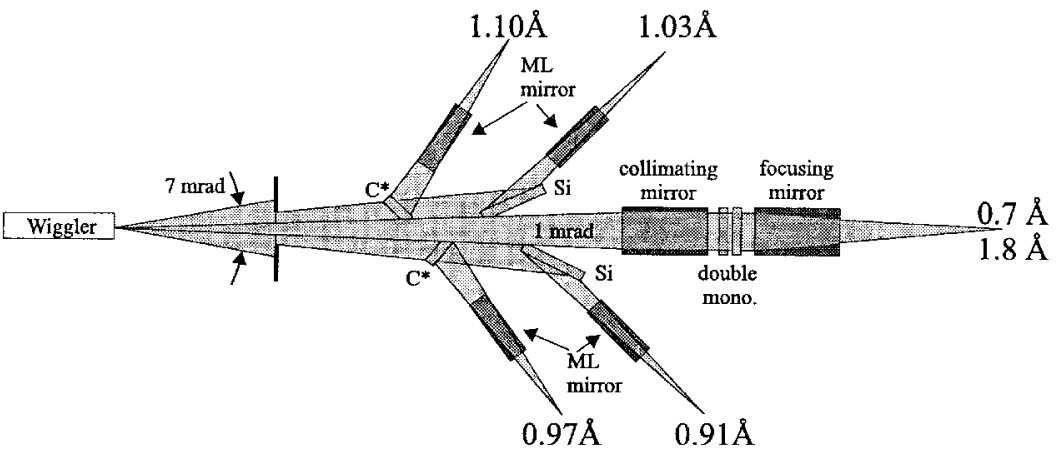

Fig. 3. Top-view sketch of the different experiment stations at the beamline. The two upstream side stations will have diamond monochromators $\left(\mathrm{C}^{*}\right)$ and the two downstream side stations silicon monochromators ( $\mathrm{Si}$ ), all being bent for horizontal focusing. All side stations will have multilayer (ML) mirrors for vertical focusing. The central, energy-tunable station will have more classical grazing incidence mirrors and a Si double crystal monochromator.

\section{TABLE II}

Predicted performance of the side stations. The monochromators of the 1.10 $\AA$ and $0.97 \AA$ stations are made of diamonds in the Laue configuration and the remaining two side stations are $\mathrm{Si}$ or Ge crystals in the Bragg configuration.

\begin{tabular}{l|l}
\hline \hline Wavelength $\lambda_{1}$ & $=1.10 \AA$ \\
Wavelength $\lambda_{2}$ & $=1.03 \AA$ \\
Wavelength $\lambda_{3}$ & $=0.91 \AA$ \\
Wavelength $\lambda_{4}$ & $=0.97 \AA$ \\
Relative bandwidth, $\Delta E / E$ & $\approx 1 \times 10^{-3}$ \\
Spot size & $\approx 0.3 \times 0.3 \mathrm{~mm}^{2}$ \\
Flux with Laue monochromator & $\approx 10^{11}$ photons $/ \mathrm{s}$ \\
Flux with Bragg monochromator & $\approx 10^{12}$ photons $/ \mathrm{s}$
\end{tabular}


TABLE III

Predicted performance of the MAD station.

\begin{tabular}{l|l}
\hline \hline Wavelength range & $=0.7 \AA$ to $1.8 \AA$ \\
Relative bandwidth, $\Delta E / E$ & $\approx 2 \times 10^{-4}$ \\
Spot size & $\approx 0.3 \times 0.25 \mathrm{~mm}^{2}$ \\
Flux at $1 \AA$ & $\approx 3 \times 10^{12}$ photons $/ \mathrm{s}$ \\
Flux at $1 \AA$ in $0.1 \times 0.1 \mathrm{~mm}^{2}$ & $\approx 5 \times 10^{11}$ photons $/ \mathrm{s}$
\end{tabular}

The optical components of the beamline will be designed and constructed according to the requirements for macromolecular crystallography. The predicted characteristics of the beam at the sample position of the four side stations are found in Table II and of the MAD station in Table III.

\section{Design of the side station optics}

The two upstream monochromators for the side stations are asymmetric diamond (111) crystals in Laue transmission geometry (Fig. 3). Diamond is chosen due to its high transmission above $10 \mathrm{keV}$ in order to allow the extraction of an additional downstream monochromatic beam. The outer dimensions of the diamond crystals will be $7 \times 7 \times 0.3 \mathrm{~mm}^{3}$, and the crystals are bent meridionally to provide focusing in the horizontal plane. This technique has previously been used with silicon crystals [19]. Additionally, the bending increases the accepted band width by a factor two. The meridional bending radius of curvature will be $12 \mathrm{~m}$ to achieve the required focusing. The fracture limit for bending of thin crystals is typically reached for $R_{\text {bent }}=1000 \cdot t$, where $t$ is the crystal thickness [20]. Hence, the required bending radii for the diamond crystals are obtainable.

The monochromators for the downstream side stations are $\mathrm{Si}(111)$ crystals in asymmetric Bragg reflection geometry. The outer dimensions are $10 \times 8 \times 0.5 \mathrm{~mm}^{3}$ and they will also be bent to provide meridional focusing. The asymmetry angle for the Si crystal at the $1.03 \AA$ station will be $2.5^{\circ}$ and its bending radius $52 \mathrm{~m}$.

The vertical focusing is obtained by curved multilayer mirrors inserted in the downstream beam from the monochromators. Multilayer mirrors are chosen due to the larger angle of beam incidence compared to conventional mirrors. This allows shorter mirrors and thus considerable cost reduction.

The four mirror substrates are identical: $450 \mathrm{~mm}$ long, made of $\mathrm{SiO}_{2}$ and polished to a fixed radius of curvature of $400 \mathrm{~m}$. The multilayer materials are alternating Si and Mo with a total of 150 bilayers. Since the angle of incidence varies along the mirror, the $d$-spacing is longitudinally graded to obtain Bragg diffraction at both ends of the mirror simultaneously. The multilayer period and longitudinal grading is specified according to the wavelengths of the individual four side stations.

The predicted characteristics of the side stations are found in Table II. 


\section{Design of the MAD station optics}

The optics of the MAD station will consist of a vertically collimating mirror, a double-crystal Si(111) monochromator and a toroidal focusing mirror. A solution where the horizontal focusing is done by the second mirror instead of by the second monochromator crystal was chosen since it was believed to give an easier-to-use beamline for the users. The collimating mirror is needed to achieve the high energy resolution (of the order of $\Delta E / E \approx 10^{-4}$ ) required by the experiments using anomalous scattering. The mirrors will be Rh-coated and operated at a fixed grazing incidence angle of $3.5 \mathrm{mrad}$ giving a smooth reflection curve in the whole accessible wavelength range of $0.7-1.8 \AA(7-18 \mathrm{keV})$ that covers the $K$-edges from $\mathrm{Fe}$ to $\mathrm{Y}$, and the $L_{\mathrm{III}}$-edges from Gd to $\mathrm{U}$. The high power of the wiggler is reduced by the relatively small angular acceptance and by windows and filters resulting in less than $200 \mathrm{~W}$ reaching the first MAD-station mirror and $160 \mathrm{~W}$ on the first monochromator crystal.

The predicted characteristics of the MAD station are found in Table III.

\section{Experiment stations}

The experiment stations will consist of relatively small radiation enclosures (approximately $1.2 \times 2.5 \mathrm{~m}^{2}$ ) with all equipment inside being reached from the outside, i.e. the experimenters will not enter into the enclosures. This saves space and also allows a more rapid interlock system. The stations will be equipped with high performance area detectors and will also be prepared for automation.

\section{References}

[1] D. Blow, Structure 8, R77 (2000).

[2] Protein Data Bank Current Holdings, http://www.rcsb.org/pdb/holdings.html.

[3] A.J. Oakley, M.C. Wilce, Clin. Exp. Pharmacol. Physiol. 27, 145 (2000).

[4] H.M. Berman, T.N. Bhat, P.E. Bourne, Z. Feng, G. Gilliland, H. Weissig, J. Westbrook, Nat. Struct. Biol. 7, 957 (2000).

[5] R.B. Russell, D.S. Eggleston, Nat. Struct. Biol. 7, 928 (2000).

[6] S. Dry, S. McCarthy, T. Harris, Nat. Struct. Biol. 7, 946 (2000).

[7] R. Bott, R. Boelens, Curr. Opin. Biotechnol. 10, 391 (1999).

[8] W. Minor, D.R. Tomchick, Z. Otwinowski, Structure 8, R105 (2000).

[9] A. Brysiak, Z. Otwinowski, W. Minor, in: Int Symp. on Synchrotron Crystallography - SYNCRYS 2001, Krynica (Poland) 2001, SYNCRYS 2001 Book of Abstracts, p. 63

[10] W.A. Hendrickson, C.M. Ogata, Methods Enzymol. 276, 494 (1997).

[11] M.A. Walsh, G. Evans, R. Sanishvili, I. Dementieva, A. Joachimiak, Acta Crystallogr. D 55, 1726 (1999). 
[12] L.M. Rice, T.N. Earnest, A.T. Brunger, Acta Crystallogr. D 56, 1413 (2000).

[13] A. Andersson, M. Eriksson, L.J. Lindgren, P. Röjsel, S. Werin, Nucl. Instrum. Methods Phys. Res. A 343, 644 (1994).

[14] Y. Cerenius, K. Ståhl, L.A. Svensson, T. Ursby, A. Oskarson, J. Albertsson, A. Liljas, J. Synchrotron Rad. 7, 203 (2000).

[15] MAX-lab, http://www.maxlab.lu.se.

[16] E. Wallén, G. LeBlanc, M. Eriksson, Nucl. Instrum. Methods Phys. Res. A 467, 118 (2001).

[17] K.-J. Kim, in: X-ray Data Booklet, Eds. A.C. Thompson, D. Vaughan, Lawrence Berkeley National Laboratory, University of California, Berkeley 2001, p. 2-1.

[18] ESRF Highlights 2000, ESRF, Grenoble, 2001, p. 100.

[19] C. Schulze, U. Lienert, M. Hanfland, M. Lorenzen, F. Zontone, J. Synchrotron Rad. 5, 77 (1998).

[20] A.K. Freund, F. Comin, J.-L. Hazemann, R. Hustache, B. Jenninger, K. Lieb, M. Pierre, in: Proc. SPIE, Vol. 3448, Eds. A.T. Macrander, A.K. Freund, T. Ishikawa, D.M. Mills, 1998, p. 144. 